\title{
Computational Method for Epidemic Detection in Multiple populations
}

\author{
Ekaterina Shatskikh* and Michael Ludkovski \\ Statistics, UC Santa Barbara, Goleta, CA, USA
}

\section{Objective}

Detect epidemics over multiple Populations using computational methods

\section{Introduction}

Currently Centers for Disease Control and Prevention (CDC) employ threshold rules to declare epidemic outbreaks, such as influenza, separately in each population. However each year influenza starts in one population and spreads population-to-population throughout the country. Therefore there is a need for an algorithm to declare the epidemic that uses information from multiple populations.

\section{Methods}

The standard stochastic SIR model can be used to simulate the epidemic behavior in one population ${ }^{1}$. We use the extended model by coupling populations with each other, where the infection can spread from one population to another.

We apply the Quickest Detection method $^{2}$ to announce the epidemic. To qualify the detection accuracy, we balance the costs of false alarms and detection delays. Thus, we will announce an epidemic if the future cost is greater than the immediate cost. Since epidemic evolution is stochastic, we generate epidemic scenarios and estimate the expected costs.

We use Sequential Regression Monte Carlo ${ }^{3}$ (SRMC) to efficiently approximate the decision rules. This consists of judiciously picking epidemic scenarios using sequential design and then applying regression techniques to construct the entire surface of expected future costs. Moreover SRMC allows the algorithm to self-correct by simulating additional epidemic scenarios for the model states where the algorithm is not sure about announcing the epidemic.

\section{Results}

The figure below illustrates our approach on a simulated dataset involving two populations of size 2000 . We constructed a detection map based on the number of infected individuals in the first population $\mathrm{I}_{\mathrm{t}}^{(1)}$ and posterior probability of epidemic in the second population $\mathrm{P}_{\mathrm{t}}$. The blue region corresponds to the regions where the epidemic should be announced; while the red region corresponds to the regions where the epidemic should not be announced. The small white regions (which can be seen on the boundary of the previous two regions) represent the regions where the algorithm is not sure about announcing the epidemic.

Our algorithm is better than the CDC approach because it uses the additional information about the number of infected individuals in other populations. This additional information helps the algorithm to see the whole picture about the state of epidemic.

\section{Conclusions}

We developed an algorithm for detecting an epidemic in a multiple population scenario. We are working on an extension of this algorithm to cover the case where we observe only partial information (for example, only diagnosed infected individuals) and we use particle filtering to estimate $\mathrm{I}_{\mathrm{t}}^{(1)}$ and $\mathrm{P}_{\mathrm{t}}$.

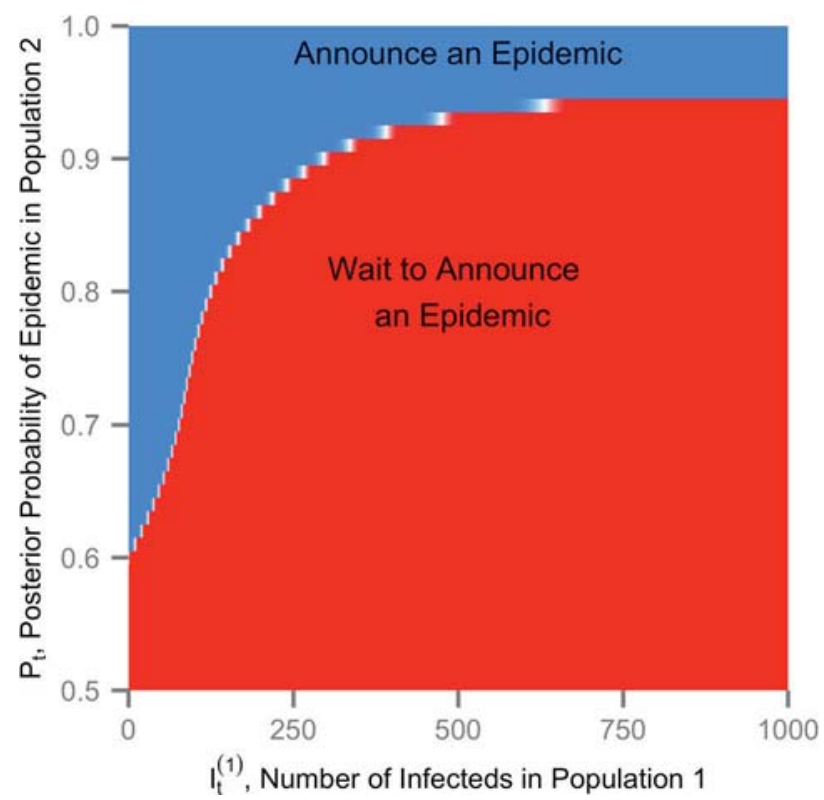

Figure: Detection map for the second population in a two-population model constructed based on 2000 adaptively generated scenarios. Decisions are based on a loess metamodel.

\section{Keywords}

Simulation; Stochastic compartmental models; Early outbreak detection

\section{Acknowledgments}

This material is based upon work partially supported by the National Science Foundation under Grant No. ATD - 1222262.

\section{References}

1. Andersson H., Britton, T. Stochastic Epidemic Models and Their Statistical Analysis. Lecture Notes in Statistics. Springer, 2000.

2. Poor H.V., Hadjiliadis O. Quickest Detection. Cambridge University Press, 2008

3. Gramacy R. Ludkovski M. Sequential Regression for Optimal Stopping Problems. arXiv:1309.3832, 2013.

*Ekaterina Shatskikh

E-mail: shatskikh@umail.ucsb.edu 